\title{
Heartbeat: Diagnosis of subclinical atrial fibrillation by physicians
} and patients

\author{
doi:10.1136/heartjnl-2019-315232
}

Catherine $\mathrm{M} \mathrm{Otto}^{\circ}$

Atrial fibrillation (AF) is estimated to cause about $15 \%$ of all strokes, yet many patients with $\mathrm{AF}$ are asymptomatic, and thus, not diagnosed or treated with preventative anticoagulation. Our ability to monitor cardiac rhythm in ambulatory patients has increased dramatically in the last few years with numerous medical and consumer devices providing continuous heart rate or ECG monitoring. In this issue of Heart, Sejr and colleagues ${ }^{1}$ compared the accuracy of AF detection by 48 hours of external loop recording (ELR) versus simultaneous continuous ECG in a prospective study of 1412 patients with an acute stroke or transient ischaemia attack. Continuous ECG documented AF in 2.7\% of patients-16\% of these patients were missed on ELR. However, ELR suggested $\mathrm{AF}$ was present in five times more patients than were documented on continuous ECG recording; cardiologist verification reduced this number but was still associated with a high number of false positive diagnoses of AF (figure 1).

In an editorial, Pokorney and Lopes ${ }^{2}$ put these data in the context of the range of devices available to medical professionals and patients (figure 2). Screening for undiagnosed $\mathrm{AF}$ is controversial on several levels-who should be screened? How often and how long should they be screened? Which device should be used? But the controversy goes beyond detection of AF; it is unclear whether there would be a favourable risk-benefit ratio for anticoagulant therapy in patients with subclinical AF. Physicians are going to need to address the treatment question quickly. After all, our patients are not going to wait for us to decide how to best screen for AF; increasingly they simply are doing it themselves.

Another important question is whether there are ethnic differences in AF prevalence. In a study of 1746 patients with heart failure (HF) by Tan and colleagues ${ }^{3}$ , AF prevalence was lower in SingaporeAsians than in New Zealand (NZ)-Europeans $(24 \%$ vs $63 \%$; $<<0.001)$. This

Correspondence to Prof. Catherine M Otto, Division of Cardiology, University of Washington, Seattle, WA 98195, USA; cmotto@uw.edu, cmotto@u.washington. edu
Flow diagram of AF diagnosis

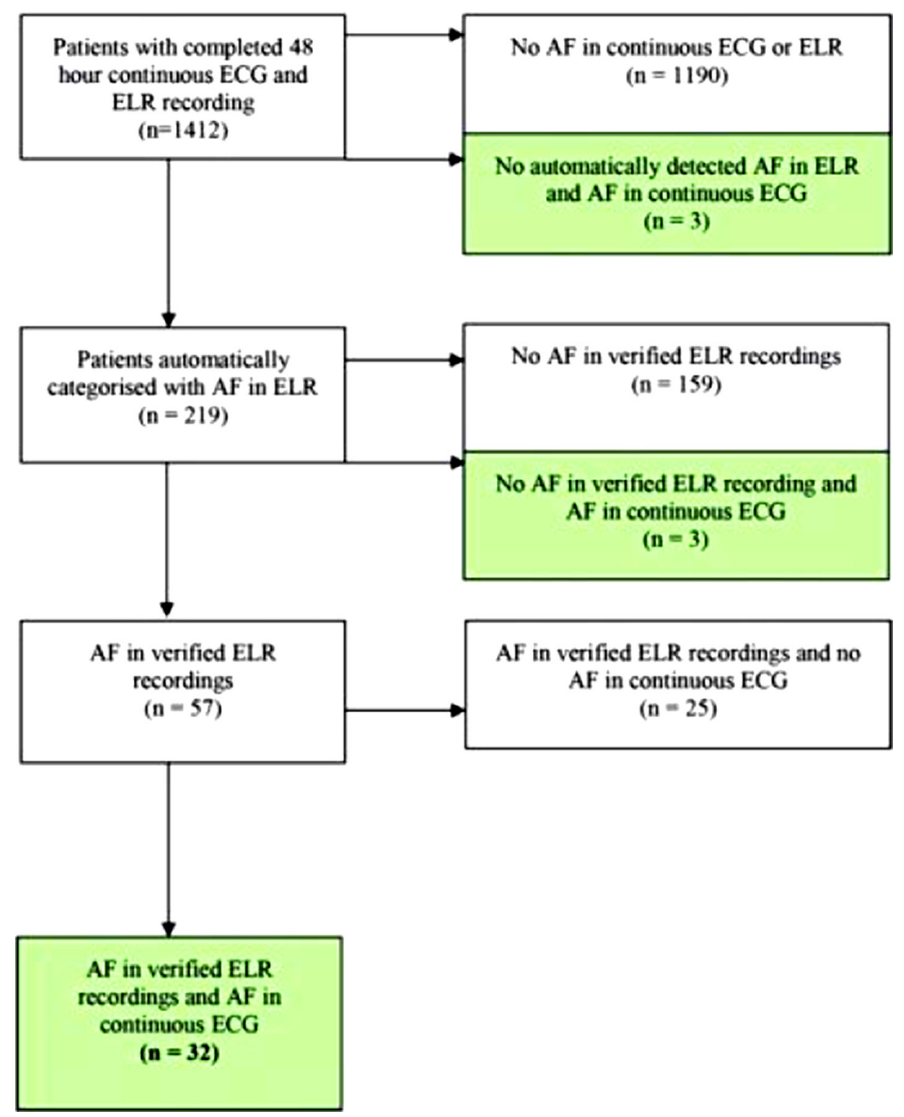

Figure 1 Flow diagram illustrating results of AF diagnostics in continuous ECG and ELR. Green marked boxes represent patients with $A F$ in continuous ECG. AF, atrial fibrillation; ELR, external loop recorder.

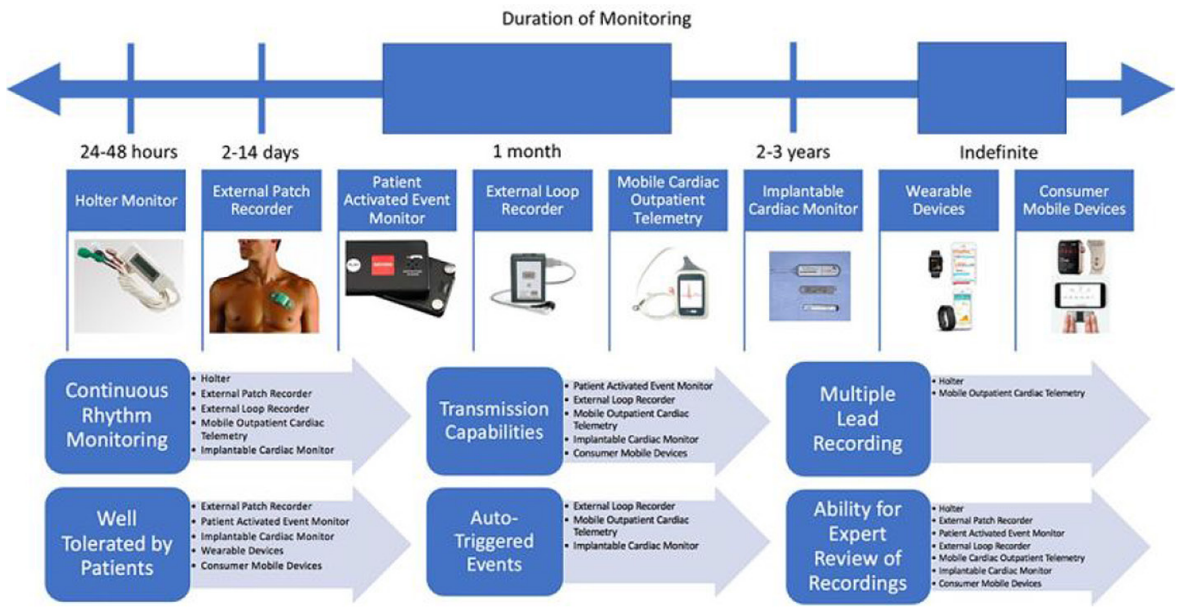

Figure 2 Screening technologies for atrial fibrillation. 


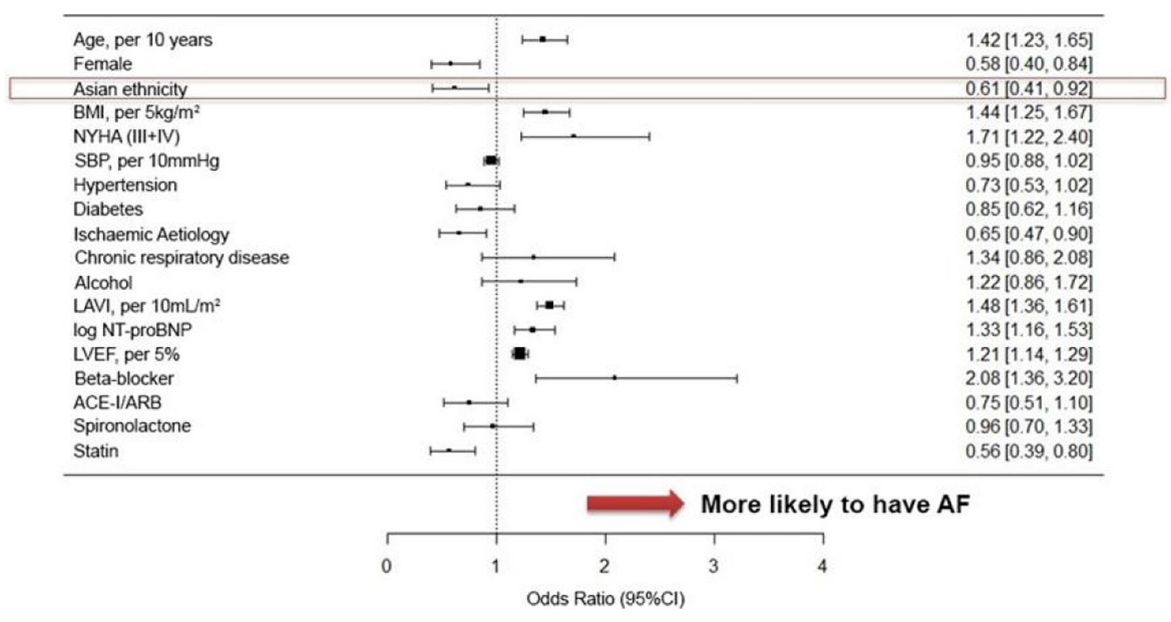

Figure 3 Adjusted OR $(95 \% \mathrm{Cl})$ for respective clinical variables and its association with $\mathrm{AF}$. $A C E-I, A C E$ inhibitor; AF, atrial fibrillation; $A R B$, angiotensin II receptor blocker; BMI, body mass index; LAVI, left atrial volume index; LVEF, left ventricular ejection fraction; NYHA, New York Heart Association; SBP, systolic blood pressure.
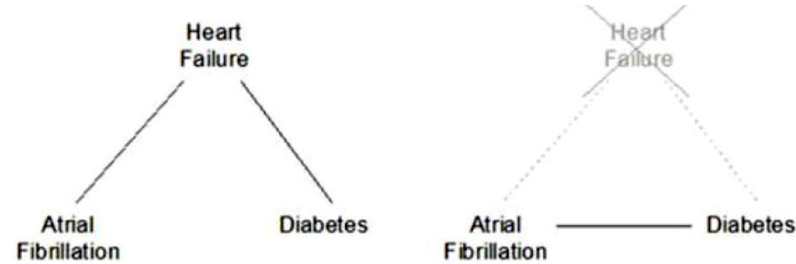

Figure 4 An illustration of the induction of associations through the removal of a collider.

1-month FU
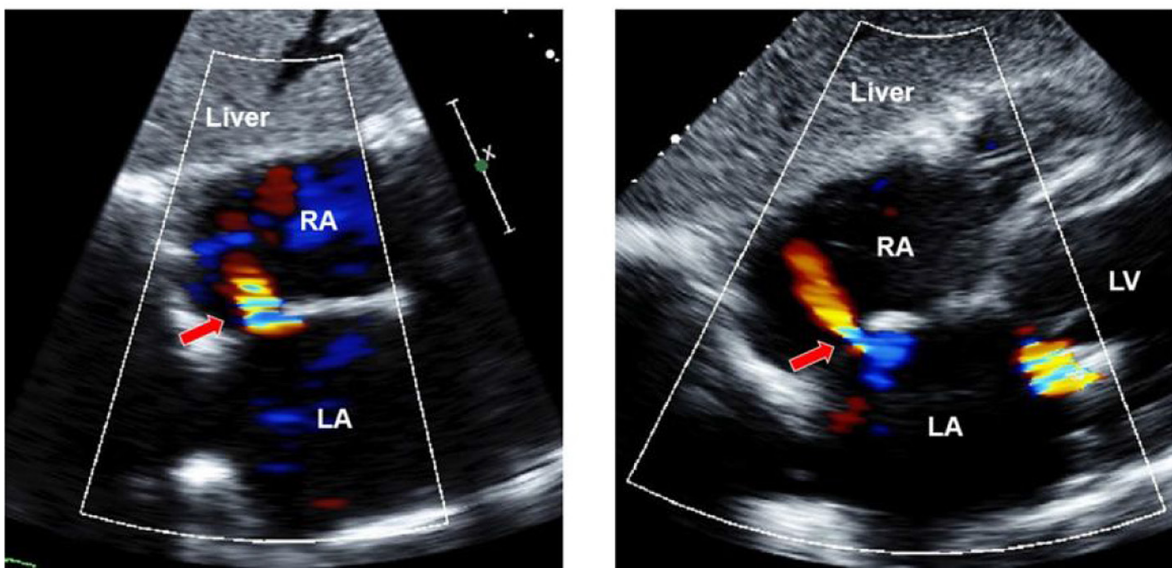

Figure 5 Examples of persistent iASD at 1 month and 12 months after MV clip procedure. Left panel is TTE image at subcostal four-chamber view at 1 month FU. Red arrow shows iASD with left to right shunt. Right panel is TTE image at 12-month FU from the same patients. Persistent iASD was still observed at 12 months (red arrow). FU, follow-up; iASD, iatrogenic atrial septal defect; LA, left atrium; LV, left ventricle; MV, mitral valve; RA, right atrium; TTE, transthoracic echocardiography.

difference persisted even after adjustment for age, relevant clinical variables and echocardiographic parameters. Comorbidities associated with AF were similar in Asians and NZ-Europeans including older age, higher body mass index, hypertension and renal disease (figure 3). However,
Asian patients with diabetes had less AF than patients without diabetes, whereas there was no difference in AF prevalence among NZ-Europeans with and without diabetes.

The accompanying editorial by Burden and Timpson ${ }^{4}$ points out that a lower prevalence of AF in Asian patients with HF is the opposite of what would be expected from prior studies which have shown that diabetes is associated with a higher risk of AF. Is this an important new finding that has clinical implications for prevention of AF or are there other possible explanations? Burden and Timpson raise the concern that collider bias might be present; specifically, that the inclusion of only patients with HF leads to an apparent relationship between two variables (ie, AF and diabetes) that are both causally related to the variable used to select the study cohort (ie, HF) (figure 4). Tan and colleagues ${ }^{5}$ respond in a letter: 'If collider bias from HF-only selection explains the diabetes-AF paradox, it is difficult to understand why collider bias would apply to Asians but not NZ-Europeans, or to preserved but not reduced ejection fraction $\mathrm{HF}$ (regardless of ethnicity), in cohorts simultaneously recruited using identical protocols. Also difficult to explain is why collider bias would not similarly impact hypertension and other HF risk factors. The diabetes-AF paradox clearly needs confirmation to establish whether it derives from collider bias or other sources.'

Other interesting papers in this issue include the study by Ikenaga and colleagues ${ }^{6}$ showing that, in patients with mitral regurgitation undergoing transcatheter edge-to-edge mitral valve repair, persistence at 12 months of the iatrogenic atrial septal defect (from the procedural transeptal puncture) is related to elevated left atrial pressure, even after adjustment for cardiac index and residual mitral regurgitation.

The 'Education in Heart' in this issue ${ }^{7}$ discusses the importance of psychosocial risk factors for coronary disease, such as social isolation and depression in addition to smoking and other behaviours. Optimising cardiovascular risk prevention includes attention to psychosocial and behavioural risk factors as detailed in practical guidance for clinicians in this article.

The 'Image Challenge' question ${ }^{8}$ shows an interesting cardiac finding on the positron emission tomography-CT scan in an elderly woman with fatigue and reduced appetite.

Funding The authors have not declared a specific grant for this research from any funding agency in the public, commercial or not-for-profit sectors.

Competing interests Not declared.

Patient consent for publication Not required. 
Provenance and peer review Commissioned; internally peer reviewed.

(c) Author(s) (or their employer(s)) 2019. No commercial re-use. See rights and permissions. Published by BMJ.

\section{D) Check for updates}

To cite Otto CM. Heart 2019;105:809-811.

Received 12 April 2019

Accepted 12 April 2019

Heart 2019;105:809-811. doi:10.1136/heartjnl-2019-315232

\section{REFERENCES}

1 Sejr MH, May O, Damgaard D, et al. External continuous ECG versus loop recording for atrial fibrillation detection in patients who had a stroke. Heart 2019;105:846-52.

2 Pokorney SD, Lopes RD. Atrial fibrillation: what do we know about screening and what do we not know about treatment? Heart 2019:105:815-7.

3 Tan ESJ, Tay WT, Teng TK, et al. Ethnic differences in atrial fibrillation in patients with heart failure from AsiaPacific. Heart 2019;105:840-5.
4 Burden AF, Timpson N. Ethnicity, heart failure, atrial fibrillation and diabetes: collider bias. Heart 2019;105:812-4.

5 Tan ESJ, Tay WT, Teng T-HK, et al. The diabetes-atrial fibrillation paradox. Heart 2019:105:890.

6 Ikenaga H, Hayashi A, Nagaura T, et al. Left atrial pressure is associated with iatrogenic atrial septal defect after mitral valve clip. Heart 2018;105:862-70

7 Astin F, Lucock M, Jennings CS. Heart and mind: behavioural cardiology demystified for the clinician. Heart 2019;105:879-85.

8 Thayaparan AS, Haikerwal D. Eighty-nine-year-old woman with fatigue and reduced appetite. Heart 2019;105:878 